\title{
Goss Orientation Evolution in Ti-5.5Mo-8Al-6Zr Shape Memory Alloy upon Heat Treatment
}

\author{
Yuri Shinohara ${ }^{1,2, * 1}$, Tomoya Sasaki ${ }^{1,2, * 2}$, Masaki Tahara ${ }^{1,2}$, Hideki Hosoda ${ }^{1,2}$ and Tomonari Inamura ${ }^{1,2}$ \\ ${ }^{1}$ Laboratory for Materials and Structures, Institute of Innovative Research, Tokyo Institute of Technology, Yokohama 226-8503, Japan \\ ${ }^{2}$ Laboratory for Future Interdisciplinary Research of Science and Technology, Institute of Innovative Research, Tokyo Institute of Technology, \\ Yokohama 226-8503, Japan
}

\begin{abstract}
The effect of heat treatment temperature on texture formation in $\mathrm{Ti}-5.5 \mathrm{Mo}-8 \mathrm{Al}-6 \mathrm{Zr}(\mathrm{mol} \%)$ alloy sheets was systematically investigated in this study. $\alpha^{\prime \prime}$ martensite was induced by cold-rolling. Although the deformation texture of $\alpha^{\prime \prime}$ martensite could not be detected, the formation of $\{130\}_{\alpha^{\prime \prime}}\langle 3 \overline{1} 0\rangle_{\alpha^{\prime \prime}}$ and $(100)_{\alpha^{\prime \prime}}[010]_{\alpha^{\prime \prime}}$ textures was proposed. The specimen heat-treated at $1073 \mathrm{~K}$ or higher consisted of a single $\beta$ phase, and Goss, $\{110\}_{\beta}\langle 113\rangle_{\beta}$, and Brass orientations were formed as the recrystallization textures. The Goss orientation, which is irregular for $\beta$-Ti alloys, developed with increasing heat treatment temperature, and became the dominant component in the specimens heat-treated at $1173 \mathrm{~K}$. The specimen heat-treated at $973 \mathrm{~K}$ consisted of the $\beta+\alpha$ phase. $\{113\}_{\beta}\langle 110\rangle_{\beta},\{113\}_{\beta}\langle 141\rangle_{\beta},\{223\}_{\beta}\langle 252\rangle_{\beta},\{223\}_{\beta}\langle 692\rangle_{\beta}$, and $\{332\}_{\beta}\langle 113\rangle_{\beta}$ were formed in the $\beta$ phase, whereas $\{11 \overline{2} 0\}_{\alpha}\langle 1 \overline{100}\rangle_{\alpha}-\{11 \overline{2} 2\}_{\alpha}\left\langle 1 \overline{100\rangle_{\alpha}}\right.$ was formed in the $\alpha$ phase. Transmission electron microscopy observations revealed that the specimen heat-treated at $973 \mathrm{~K}$ was not completely recrystallized. This microstructural difference led to a difference in texture components between the specimens heat-treated at $973 \mathrm{~K}$ and at $1073 \mathrm{~K}$ or higher. [doi:10.2320/matertrans.M2019039]
\end{abstract}

(Received February 12, 2019; Accepted May 22, 2019; Published June 21, 2019)

Keywords: titanium alloys, texture, Goss orientation, shape memory alloys, superelasticity, metallic biomaterials

\section{Introduction}

$\beta$-Ti alloys are widely used to develop biomaterials because of their high biocompatibility, corrosion resistance, and cold workability. ${ }^{1,2)}$ In particular, these alloys are promising alternatives to $\mathrm{Ti}-\mathrm{Ni}$ shape memory alloys (SMAs), which are associated with the risk of Ni hypersensitivity despite their widespread use in the biomedical field. ${ }^{3)}$ The shape memory effect and superelasticity of $\beta$-Ti alloys originate from martensitic transformation from $\beta$ (bcc) to $\alpha^{\prime \prime}$ martensite (c-orthorhombic) and their reverse transformation. Lattice deformation strain (LDS), one of the factors that determine the shape recovery strain of SMAs, depends on the crystallographic parameters (e.g. lattice parameters and crystal direction). The shape memory properties of $\beta$-Ti alloys can be improved by controlling the texture, and in this regard, the relationship between the texture and shape memory properties have been investigated extensively. ${ }^{4-11)}$ The $\{001\}_{\beta}\langle 110\rangle_{\beta},\{112\}_{\beta}\langle 110\rangle_{\beta}$, $\{111\}_{\beta}\langle 110\rangle_{\beta}$, and $\{111\}_{\beta}\langle 112\rangle_{\beta}$ orientations are formed as rolling or recrystallization textures in $\beta$-Ti alloy sheets (the subscripts indicate the phase) ${ }^{4-14)}$ The rolling direction (RD) is parallel to the $\langle 110\rangle_{\beta}$ or $\langle 112\rangle_{\beta}$ directions in these textures. In particular, under tensile stress, $\beta$-Ti SMAs preferentially adopt the $\langle 110\rangle_{\beta} / / \mathrm{RD}$ direction. It is expected that large shape recovery stain is obtained along this direction because the maximum tensile component of LDS can be obtained along $\langle 110\rangle_{\beta}{ }^{6,15-20)}$ However, the compressive component of the LDS is minimum along $\langle 110\rangle_{\beta}{ }^{21)}$ For practical application, it is desirable that the tensile and compressive components of the LDS are large and close to each other.

We have previously reported that Goss orientation, which is irregular for $\beta$-Ti alloys, is formed in Ti-Mo-Al-Zr SMAs

\footnotetext{
${ }^{* 1}$ Corresponding author, E-mail: shinohara.y.aa@m.titech.ac.jp

${ }^{* 2}$ Graduate Student, Tokyo Institute of Technology
}

sheets. $^{22-24)}$ It is reported that the Goss orientation is formed in some $\beta$-Ti alloys, though it is not dominant. ${ }^{25)}$ In contrast, this is the dominant texture component in $\mathrm{Ti}-\mathrm{Mo}-\mathrm{Al}-\mathrm{Zr}$ alloys. The Goss orientation, $\{110\}_{\beta}\langle 001\rangle_{\beta}$, is $\langle 001\rangle_{\beta} / / \mathrm{RD}$ and this orientation is preferential direction when compressive stress is applied to $\beta$-Ti SMAs because the maximum LDS of compressive component can be obtained along $\langle 001\rangle_{\beta}{ }^{21,26)}$ On the other hand, the minimum tensile component of the LDS is obtained along the $\langle 111\rangle_{\beta}$ direction and not along the $\langle 001\rangle_{\beta}$ direction, ${ }^{6,15-19)}$ indicating that the tensile and compressive components of the LDS are balanced better along $\langle 001\rangle_{\beta}$ than along $\langle 110\rangle_{\beta}$.

In addition, the formation of the $\langle 001\rangle_{\beta} / / \mathrm{RD}$ texture is useful for obtaining an implant material with low Young's modulus. Young's modulus around $30 \mathrm{GPa}$ is required for implant material because differences in Young's modulus of implants and bone lead to bone resorption. ${ }^{2)}$ In $\beta$-Ti alloys, Young's modulus shows a large anisotropy and assumes the minimum value along $\langle 001\rangle_{\beta},{ }^{27-29)}$ thus the formation of the $\langle 001\rangle_{\beta} / / \mathrm{RD}$ texture is effective to reduce Young's modulus along the RD. Indeed, Young's modulus along the $\mathrm{RD}$ decreased in sheets with Goss orientation and in coldgroove-rolled wires with $\langle 001\rangle_{\beta}$-fiber. ${ }^{24,28)}$

With this background, we state that the formation of Goss orientation is advantageous for biomedical SMAs and implant materials. In our previous study, we investigated the effect of thermomechanical treatment on texture formation in Ti-5.5Mo-8Al-6Zr (mol\%) alloy sheets. ${ }^{22,23)}$ The following aspects were revealed: (1) $\alpha^{\prime \prime}$ martensite is induced by severe cold-rolling and retained after cold-rolling, (2) Goss orientation is formed in the sheet subjected to severe coldrolling (nominal reduction in thickness is higher than 90\%) and subsequent heat treatment at $1173 \mathrm{~K}$. However, the formation conditions for the Goss orientation have not been clarified. Hence, in the present study, the effect of heat treatment conditions on the texture evolution and micro- 
structure formation in Ti-5.5Mo-8Al-6Zr shape memory alloy was systematically investigated.

\section{Experimental}

\subsection{Specimen preparation}

An alloy with the composition Ti-5.5Mo-8Al-6Zr (mol\%) was fabricated by Ar-arc melting in an $\mathrm{Ar}-1 \% \mathrm{H}_{2}$ atmosphere and homogenized at $1273 \mathrm{~K}$ for $7.2 \mathrm{ks}$. The obtained ingot was cold-rolled without lubricant with nominal reduction in thickness to $99 \%$ (final thickness of the sheet was about $250 \mu \mathrm{m})$. The test specimens were heat-treated at 973, 1073, and $1173 \mathrm{~K}$ for $3.6 \mathrm{ks}$ and labeled based on the thermomechanical process used. For example, "CR" denotes the coldrolled specimen and "HT1173" denotes the "CR" specimen heat-treated at $1173 \mathrm{~K}$. All heat-treatment processes (homogenization and heat-treatment) were performed in an $\mathrm{Ar}$ atmosphere, and the heat-treated samples were quenched in water.

\subsection{Phase constituents, texture measurements, and microstructure observation}

All measurements were performed at room temperature. The phase constituents and lattice parameters were identified by X-ray diffraction (XRD) analysis using a $\mathrm{Cu}-\mathrm{K} \alpha \mathrm{X}$-ray source. An X-ray diffractometer equipped with a onedimensional detector (X'pert MPD, Malvern Panalytical) was used.

The texture in the CR specimen was evaluated by X-ray pole figure (XPF) measurements. The global and local textures in the HT specimens were evaluated by XPF and electron backscatter diffraction (EBSD) measurements, respectively. The XPF measurements were performed using an X-ray diffractometer with a $\mathrm{Cu}-\mathrm{K} \alpha \mathrm{X}$-ray source (X'pert MRD, Malvern Panalytical). A polycapillary X-ray lens and a parallel plate collimator were used on the incident and detector sides, respectively. The specimen size was set to $8 \mathrm{~mm} \times 8 \mathrm{~mm}$, and the elevation angle was varied from $0^{\circ}$ to $80^{\circ}$. The maximum beam size of the X-rays for these measurements was approximately $30 \mathrm{~mm} \times 1 \mathrm{~mm}$, and therefore, the specimen size was much smaller than the beam size. The orientation distribution functions (ODFs) were constructed based on the measured XPFs using a texture analysis software program (X'pert texture, Malvern Panalytical). The algorithm used in this program is the Williams-ImhofMatthies-Vinel (WIMV) method. Pole figures for ODF calculations were as follows: $110_{\beta}, 211_{\beta}, 222_{\beta}$, and $310_{\beta}$ for $\beta$ phase in HT1173 and HT1073; $200_{\beta}$ and $310_{\beta}$ for $\beta$ phase in HT973; and $1 \overline{1} 00_{\alpha}, 1 \overline{1} 02_{\alpha}$, and $\overline{2} 110_{\alpha}$ for $\alpha$ phase in HT973.

EBSD analysis was performed using a scanning electron microscope (SU5000, Hitachi) equipped with an EBSD detector. The observation area was $3 \times 10^{-4} \mathrm{~mm}^{2}$ for HT973, and $5 \mathrm{~mm}^{2}$ for HT1073 and HT1173. An orientation imaging microscopy system (OIM, TSL Solutions) was used to analyze the inverse pole figure (IPF) maps, grain reference orientation deviation (GROD) maps and calculate the ODFs. The ODFs were calculated using a harmonic series expansion. The results of EBSD analysis were used for a quantitative texture analysis. The texture strength was evaluated based on the orientation density in these ODFs. Grain boundaries were defined by a misorientation of $2^{\circ}$ or more. The specimens for the XPF and EBSD measurements were finished by electropolishing in a mixture of $6 \mathrm{vol} \%$ perchloric acid, $35 \mathrm{vol} \%$ butanol, and $59 \mathrm{vol} \%$ methanol at $233 \mathrm{~K}$. Measurement was performed at $50-\mu \mathrm{m}$ depth from the rolled surface.

The microstructure of HT973 was evaluated by transmission electron microscopy (TEM, JEOL, JEM-2100) at $200 \mathrm{kV}$. The specimens for TEM observations were finished by twin-jet polishing in a mixture of $5 \mathrm{vol} \%$ sulfuric acid, 2 vol\% hydrofluoric acid, and 93 vol\% methanol at $233 \mathrm{~K}$.

\section{Results and Discussion}

\subsection{Phase constituents}

Figure 1 shows the XRD profiles of the CR and HT1173HT973 specimens. $\alpha^{\prime \prime}$ martensite was detected in the CR specimen, but it could not be discerned whether the peaks at $2 \theta=38^{\circ}$ and $2 \theta=82^{\circ}$ were due to the $\alpha^{\prime \prime}$ phase or $\beta$ phase. It has been reported that $\alpha^{\prime \prime}$ martensite is induced by severe deformation and that it remains in $\mathrm{Ti}-5.5 \mathrm{Mo}-8 \mathrm{Al}-6 \mathrm{Zr}$ alloy sheets. $^{22,23)}$ In other words, stress-induced martensitic transformation (SIMT) occurs during cold-rolling. Thus, the phase constituents identified in this study were consistent with the literature data. The lattice parameters of the $\alpha^{\prime \prime}$ phase

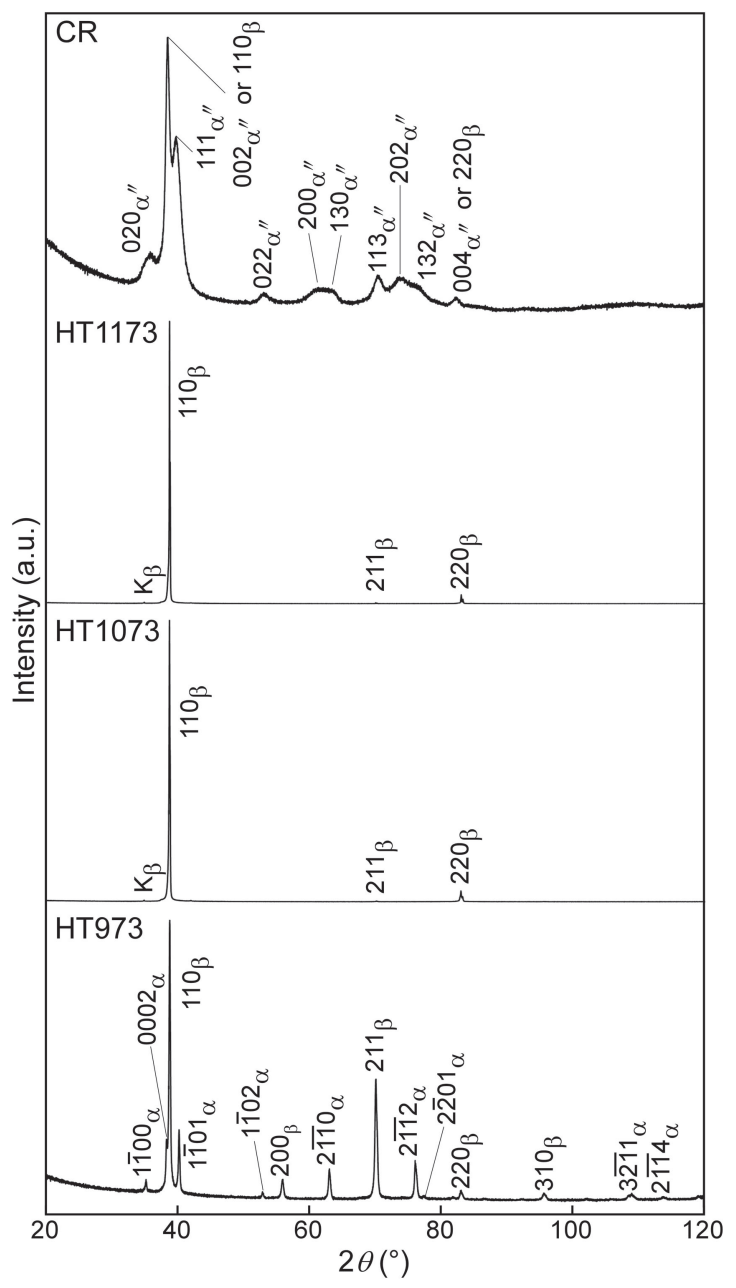

Fig. 1 XRD profiles for CR, HT1173, HT1073, and HT973 specimens. 


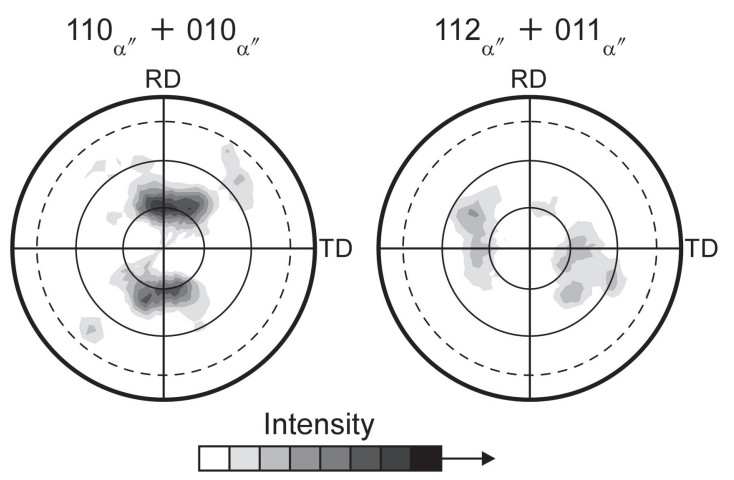

Fig. 2 PFs for a CR specimen measured at $2 \theta=35.7^{\circ}$ and $53.1^{\circ}$. Broken line indicates the limit of the measurement range.

determined from this XRD profile were $a_{\alpha^{\prime \prime}}=0.3038 \mathrm{~nm}$, $b_{\alpha^{\prime \prime}}=0.5040 \mathrm{~nm}$, and $c_{\alpha^{\prime \prime}}=0.4684 \mathrm{~nm}$. However, these values likely have large errors because of peak broadening owing to the introduction of dislocations.

In contrast, sharp peaks diffracted from the $\beta$ phase were observed in HT1173-HT1073, whereas peaks due to the $\alpha$ (hcp) and $\beta$ phase were observed in HT973. The fact that only the $\beta$ phase was observed in the specimens heat-treated at temperatures of $1073 \mathrm{~K}$ or higher implied reverse transformation of the $\alpha^{\prime \prime}$ phase to the $\beta$ phase under these conditions as well as the existence of $\beta$-transus between 973 and $1073 \mathrm{~K}$. The estimated $\beta$-transus of this alloy using the equation proposed by Guo et al. was $1072 \mathrm{~K} .{ }^{30}$ ) The lattice parameter of the $\beta$ phase was $a_{\beta}=0.3290 \mathrm{~nm}$, as calculated from the HT1173 profile.

\subsection{Textures of the CR specimen}

Figure 2 shows the XPFs of the $\alpha^{\prime \prime}$ phase in the CR specimen under the conditions $2 \theta=35.7^{\circ}$ and $53.1^{\circ}$, which correspond to the diffraction angles of $020_{\alpha^{\prime \prime}}$ and $022_{\alpha^{\prime \prime}}$ in Fig. 1. However, other poles with similar plane spacing could overlap with these PFs because the plane-spacing ranges were broadened due to the introduction of dislocations in the CR specimens. Lattice parameters obtained in Section 3.1 indicate that $110_{\alpha^{\prime \prime}}\left(2 \theta=34.5^{\circ}\right)$ and $112_{\alpha^{\prime \prime}}\left(2 \theta=52.5^{\circ}\right)$ peaks exist in the vicinity of $020_{\alpha^{\prime \prime}}$ and $022_{\alpha^{\prime \prime}}$ peaks. Thus, it is reasonable to assume that Fig. 2 indicates $110_{\alpha^{\prime \prime}}+010_{\alpha^{\prime \prime}}$ and $112_{\alpha^{\prime \prime}}+011_{\alpha^{\prime \prime}}$ PFs. The $110_{\alpha^{\prime \prime}}$ and $010_{\alpha^{\prime \prime}}$ planes are tilted by about $30^{\circ}$ toward the RD from the ND, while the $112_{\alpha^{\prime \prime}}$ and $011_{\alpha^{\prime \prime}}$ planes are tilted by $30^{\circ}-60^{\circ}$ from the ND.

On the basis of the aforementioned results, we attempted to identify the textures satisfy the following conditions for $010_{\alpha^{\prime \prime}}$ and $011_{\alpha^{\prime \prime}}$ poles. The conditions for the $010_{\alpha^{\prime \prime}}$ poles are as follows: the poles are tilted by about $30^{\circ}$ toward the RD from the ND, or are out of the measurement range (tilted by more than $80^{\circ}$ from the ND). The conditions for the $011_{\alpha^{\prime \prime}}$ poles are as follows: the poles are tilted by $30^{\circ}-60^{\circ}$ from the ND, or are out of the measurement range. Table 1 shows some candidate orientations that satisfy these conditions.

We could not identify the orientation of the deformation texture because if the large number of candidates, such as $\{130\}_{\alpha^{\prime \prime}}\langle 3 \overline{1} 0\rangle_{\alpha^{\prime \prime}}$ and $\{301\}_{\alpha^{\prime \prime}}-(100)_{\alpha^{\prime \prime}} / /$ ND. However, we focused on two orientations, $\{130\}_{\alpha^{\prime \prime}}\langle 310\rangle_{\alpha^{\prime \prime}}$ and $(100)_{\alpha^{\prime \prime}}[010]_{\alpha^{\prime \prime}}$. The ideal position of the $110_{\alpha^{\prime \prime}}+010_{\alpha^{\prime \prime}}$ and $112_{\alpha^{\prime \prime}}+011_{\alpha^{\prime \prime}}$ poles in these orientations is shown in Fig. 3.
Table 1 List of candidate textures in the CR specimen. The condition satisfying the orientation is also shown (A: tilted by about $30^{\circ}$ toward the $\mathrm{RD}$ from the $\mathrm{ND}, \mathrm{B}$ : tilted by $30^{\circ}-60^{\circ}$ from the ND, C: out of the measurement range).

\begin{tabular}{lcc}
\hline & \multicolumn{2}{c}{ Condition } \\
& $010_{\alpha^{\prime \prime}}$ & $011_{\alpha^{\prime \prime}}$ \\
\hline$\{130\}_{\alpha^{\prime \prime}}<3 \overline{1} 0>_{\alpha^{\prime \prime}}$ & $\mathrm{A}$ & $\mathrm{B}$ \\
\hline$\{h 01\}_{\alpha^{\prime \prime}}-(100)_{\alpha^{\prime \prime}} / / \mathrm{ND}$ & & \\
$h \geq 3$ & $\mathrm{C}$ & $\mathrm{C}$ \\
\hline$\{h 10\}_{\alpha^{\prime \prime}}-(100)_{\alpha^{\prime \prime}} / / \mathrm{ND}$ & $\mathrm{C}$ & $\mathrm{C}$ \\
$h \geq 4$ & & \\
\hline$\{10 l\}_{\alpha^{\prime \prime}}-(001)_{\alpha^{\prime \prime}} / / \mathrm{ND}$ & & \\
$l \geq 2$ & $\mathrm{C}$ & $\mathrm{B}$ \\
\hline
\end{tabular}

(a) $\{130\}_{\alpha^{\prime \prime}}<3 \overline{1} 0>_{\alpha^{\prime \prime}}$

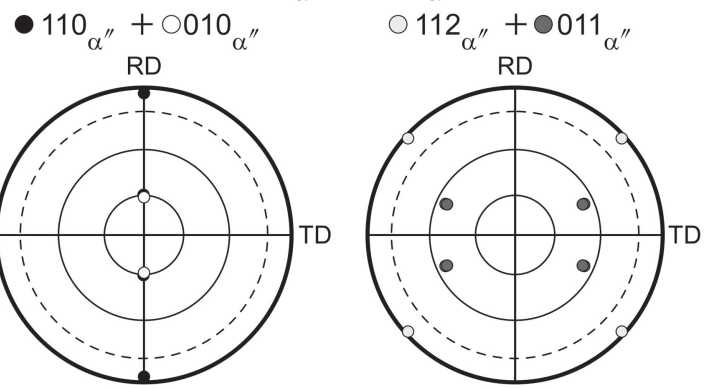

(b) $(100)_{\alpha^{\prime \prime}}[010]_{\alpha^{\prime \prime}}$

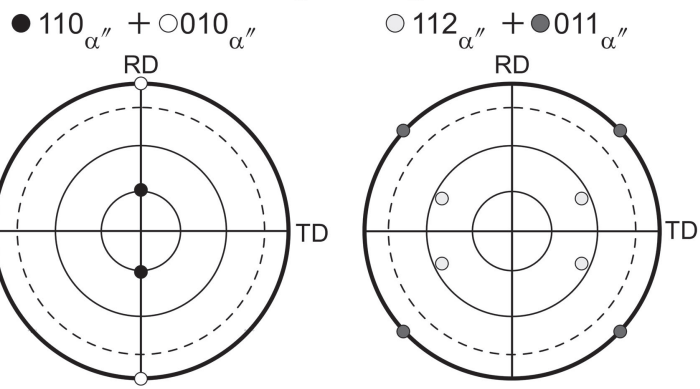

Fig. 3 Ideal position of poles in (a) $\{130\}_{\alpha^{\prime \prime}}\langle 3 \overline{1} 0\rangle_{\alpha^{\prime \prime}}$, (b) $(100)_{\alpha^{\prime \prime}}[010]_{\alpha^{\prime \prime}}$ Broken lines indicate the limit of the measurement range. In Fig. 3(a), $010_{\alpha^{\prime \prime}}$ poles overlap with $110_{\alpha^{\prime \prime}}$ at the position tilted by about $30^{\circ}$ toward the RD from the ND, and $011_{\alpha^{\prime \prime}}$ poles overlap with $112_{\alpha^{\prime \prime}}$ at the position tilted by about $55^{\circ}$ from the ND.

Only the $010_{\alpha^{\prime \prime}}$ poles in $\{130\}_{\alpha^{\prime \prime}}\langle 3 \overline{1} 0\rangle_{\alpha^{\prime \prime}}$ are tilted by about $30^{\circ}$ toward the $\mathrm{RD}$ from the ND, while this pole in the other orientations listed in Table 1 are located out of the measurement range. The position of the $110_{\alpha^{\prime \prime}}$ and $112_{\alpha^{\prime \prime}}$ poles in $(100)_{\alpha^{\prime \prime}}[010]_{\alpha^{\prime \prime}}$ correspond to the experimental data, though the $010_{\alpha^{\prime \prime}}$ and $011_{\alpha^{\prime \prime}}$ poles in this orientation are out of the measurement range. $(100)_{\alpha^{\prime \prime}}[010]_{\alpha^{\prime \prime}}$ corresponds to the 

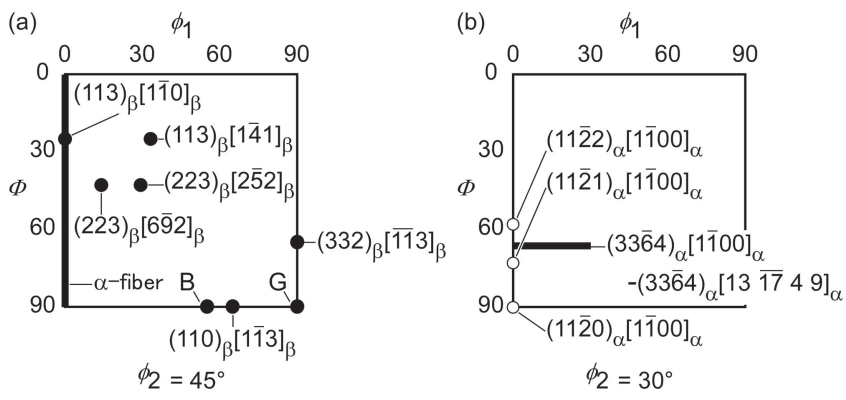

Fig. 4 Some key orientations in ODFs at (a) $\phi_{2}=45^{\circ}$ for the $\beta$ phase and (b) $\phi_{2}=30^{\circ}$ for the $\alpha$ phase. G and B represent Goss and Brass orientation, respectively.

$\langle 110\rangle_{\beta} / / \mathrm{RD}$ texture ( $\alpha$-fiber) which has commonly been reported as a deformation texture in bcc alloys ${ }^{31)}$ when the lattice correspondence of the martensitic transformation is considered.

\subsection{Textures of HT specimens}

Figure 4 shows the locations of the key orientations and fiber in the $\phi_{2}=45^{\circ}$ (for $\beta$ phase) and $\phi_{2}=30^{\circ}$ (for $\alpha$ phase) cross section of the ODF. In the $\alpha$ phase, $\left\{\phi_{1}, \Phi, \phi_{2}\right\}$ is set to $(0001)_{\alpha}[2 \overline{1} \overline{1} 0]_{\alpha}$. Hereafter, the ODF coordinates follow those in Fig. 4. The $\phi_{2}=45^{\circ}$ cross section of the ODFs in the $\beta$ phase for HT1173-HT973 is shown in Fig. 5. The $\alpha$ phase is detected in HT973, so the $\phi_{2}=30^{\circ}$ cross section of the ODF in the $\alpha$ phase for HT973 is also shown in Fig. 5. The left and right columns indicate the ODFs calculated from the information obtained in XPF (XPF-ODF) and EBSD (EBSD-ODF) measurements, respectively. The XPF-ODFs and EBSD-ODFs indicate the global and local texture.

For the global texture, texture with a maximum intensity close to $\{110\}_{\beta}\langle 001\rangle_{\beta}$ (Goss orientation) developing along $\Phi=90^{\circ}$ line on the $\phi_{2}=45^{\circ}$ sections was observed in HT1173 and HT1073. $\alpha$-fiber, $\langle 110\rangle_{\beta} / /$ RD, which are commonly formed in $\beta$-Ti alloys were not observed in these specimens.

On the other hand, $\{113\}_{\beta}\langle 110\rangle_{\beta},\{113\}_{\beta}\langle 141\rangle_{\beta}$, $\{223\}_{\beta}\langle 252\rangle_{\beta},\{223\}_{\beta}\langle 692\rangle_{\beta}$, and $\{332\}_{\beta}\langle 113\rangle_{\beta}$ were observed in HT973. $\{113\}_{\beta}\langle 110\rangle_{\beta}$ were included in the $\alpha$-fiber, while Goss, $\{110\}_{\beta}\langle 113\rangle_{\beta}$, and Brass orientations disappeared. The $\alpha$ phase in HT973 formed $\{11 \overline{2} 0\}_{\alpha}\langle 1 \overline{1} 00\rangle_{\alpha}-$ $\{11 \overline{2} 2\}_{\alpha}\langle 1 \overline{1} 00\rangle_{\alpha}$. The texture of the $\alpha$ phase partially corresponds to that of the $\beta$-phase in HT973 when the Burgers orientation relationship between the two phases is considered. ${ }^{32)}(233)_{\beta}[3 \overline{1} \overline{1}]_{\beta}$ corresponds to $(11 \overline{2} 0)_{\alpha}[\overline{1} 100]_{\alpha}$. $(233)_{\beta}[3 \overline{1} \overline{1}]_{\beta}$ also corresponds to the deformation texture, $(130)_{\alpha^{\prime \prime}}[3 \overline{1} 0]_{\alpha^{\prime \prime}}$, listed in Table 1 when the lattice correspondence of the martensitic transformation is considered. This correspondence among $\alpha, \alpha^{\prime \prime}$, and $\beta$ is depicted in Fig. 6 . The orthorhombic lattices in these three phases is marked in thick lines; the hep structure can be regarded as having an orthorhombic lattice. Based on this orientation relationship, orientation spread from $(\overline{3} 1 \overline{1})_{\beta}[011]_{\beta}$ to $(\overline{3} 1 \overline{1})_{\beta}[141]_{\beta}$ observed in Fig. 5(a) corresponds to $(6 \overline{3} \overline{3} \overline{4})_{\alpha}[0110]_{\alpha}-$

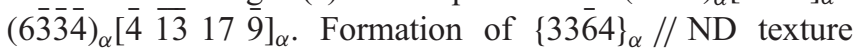
was confirmed in the ODF of $\alpha$ phase.

As shown in Fig. 5, the global texture component of HT1173 and HT1073 is reflected in the local texture. Texture

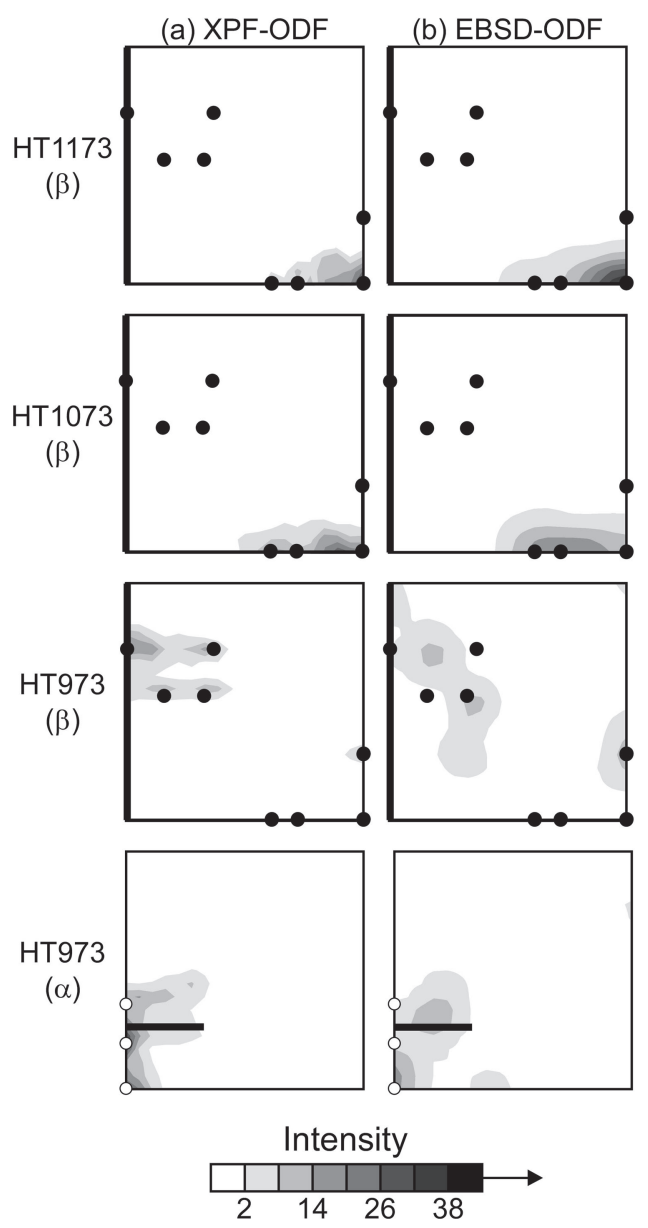

Fig. 5 Textures in HT specimens (a) global textures (XPF-ODFs), (b) local textures (EBSD-ODFs). Value of texture intensity was obtained from EBSD-ODFs. The ODFs coordinates follow those in Fig. 4.

with a maximum intensity close to the Goss orientation also appears in the EBSD-ODFs. Figure 7 shows the intensity profile along the $\Phi=90^{\circ}$ line on the $\phi_{2}=45^{\circ}$ sections of EBSD-ODF in HT1173 and HT1073. $\{110\}_{\beta}\langle 112\rangle_{\beta}$ (Brass orientation) slightly developed in addition to Goss orientation in HT1173, whereas texture with a maximum intensity at $\{110\}_{\beta}\langle 113\rangle_{\beta}$ was observed in HT1073. However, the EBSDODF in the $\beta$ phase of HT973 has little in common with that of the XPF-ODF, although the EBSD-ODF for the $\alpha$ phase seems consistent with that of the XPF-ODF. The formation of $\{113\}_{\beta}\langle 110\rangle_{\beta},\{223\}_{\beta}\langle 252\rangle_{\beta}$, and $\{332\}_{\beta}\langle 113\rangle_{\beta}$ in the XPFODF is reflected in the EBSD-ODF, although the intensity of $\{113\}_{\beta}\langle 141\rangle_{\beta}$ and $\{223\}_{\beta}\langle 692\rangle_{\beta}$ are not reflected in the EBSD-ODF. As the observation area for HT973 was too small, the data obtained from EBSD did not correspond to the entire specimen; the observation area for HT973 was extremely small as compared to that for HT1073 and HT1173, as mentioned in section 2.2. For quantitative analysis, we regard the local texture information as that for the global texture of HT1173 and HT1073. In addition, the texture components confirmed in both the XPF-ODF and EBSD-ODF $\quad\left(\{223\}_{\beta}\langle 252\rangle_{\beta}, \quad\{332\}_{\beta}\langle 113\rangle_{\beta}\right.$, and $\left.\{113\}_{\beta}\langle 110\rangle_{\beta}\right)$ are discussed for HT973.

Figures 8(a) and (b) show the relationship between the heat treatment temperature and the texture intensity in the ODFs. The texture components formed in HT1173 and 

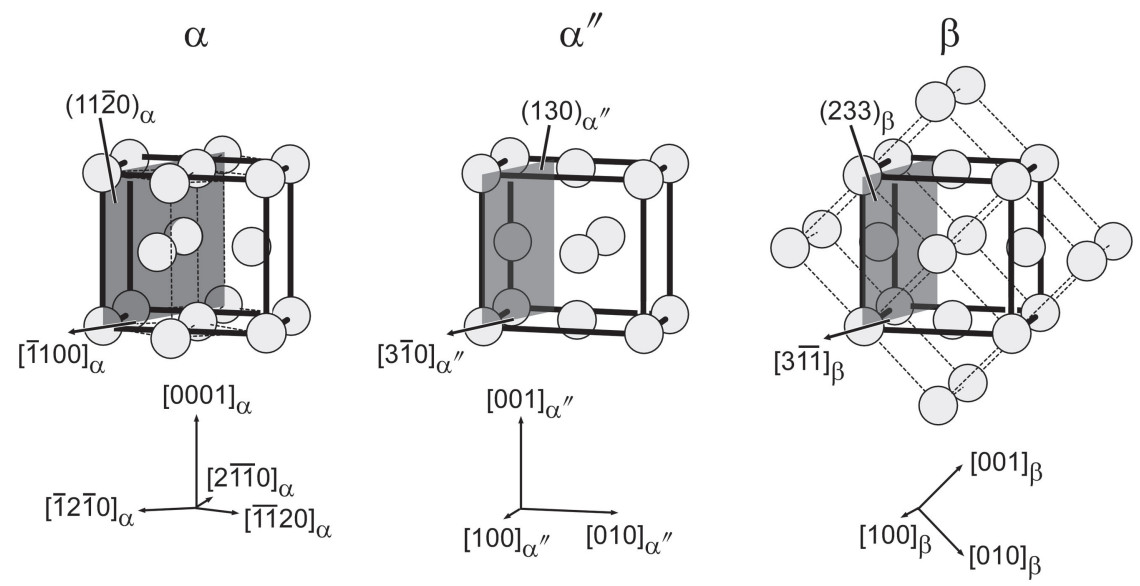

Fig. 6 Correspondence among $(11 \overline{2} 0)_{\alpha}[\overline{1} 100]_{\alpha},(130)_{\alpha^{\prime \prime}}[3 \overline{1} 0]_{\alpha^{\prime \prime}}$, and $(233)_{\beta}[3 \overline{1} \overline{1}]_{\beta}$. Orthorhombic lattices are marked in thick lines.

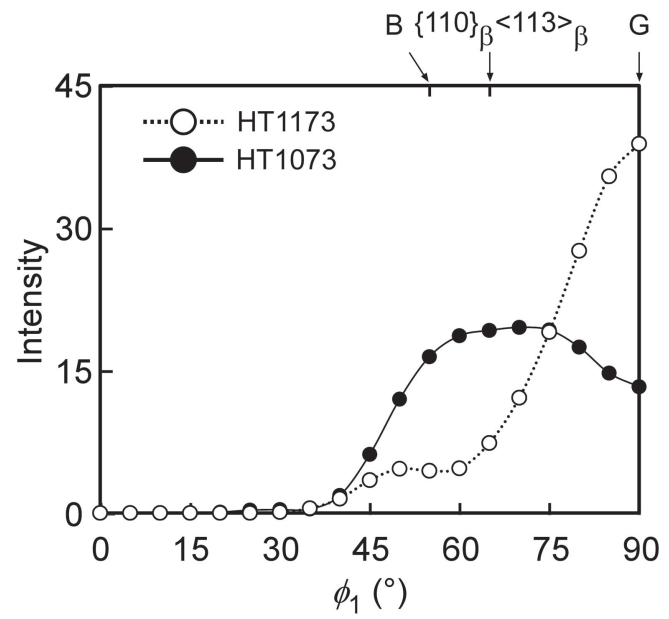

Fig. 7 Intensity profiles along the $\Phi=90^{\circ}$ line on $\phi_{2}=45^{\circ}$ sections of ODF for HT1173 and HT1073 specimens. G and B represent Goss and Brass orientation, respectively.

HT1073 are shown in Fig. 8(a), namely, Goss, $\{110\}_{\beta}\langle 113\rangle_{\beta}$, and Brass orientations. The Goss orientation is dominant in HT1173, while the intensity difference among these three orientations is small in HT1073. In other words, the Goss orientation strongly develops with increasing heat treatment temperature, with the maximum texture intensity of 38 in HT1173. The intensity of the $\{110\}_{\beta}\langle 113\rangle_{\beta}$ and Brass orientations is the highest in HT1073. It is noted that the intensity of the Goss, $\{110\}_{\beta}\langle 113\rangle_{\beta}$, and Brass orientations is close to zero in HT973.

The texture components formed in HT973, $\{113\}_{\beta}\langle 110\rangle_{\beta}$, $\{223\}_{\beta}\langle 252\rangle_{\beta}$, and $\{332\}_{\beta}\langle 113\rangle_{\beta}$ are shown in Fig. $8(\mathrm{~b})$. These orientations are formed only in HT973 but not in HT1173 and HT1073 (intensity was near zero). The texture component dramatically switched when the heat treatment temperature is decreased from $1073 \mathrm{~K}$ to $973 \mathrm{~K}$.

\subsection{Microstructure of HT specimens}

Figure 9 shows the IPF (ND) maps and GROD maps of the $\beta$ phase for HT1173-HT973 and the $\alpha$ phase for HT973. Equiaxed recrystallized grains of the $\beta$ phase were observed in HT1173 and HT1073. On the other hand, a duplex microstructure of the $\beta$ and $\alpha$ phase was observed in HT973. The grain size and area fraction of the $\alpha$ phase in HT973 were (a)

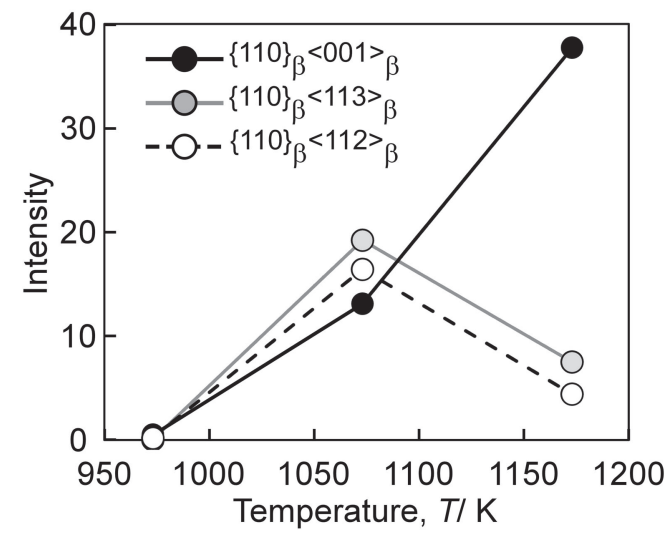

(b)

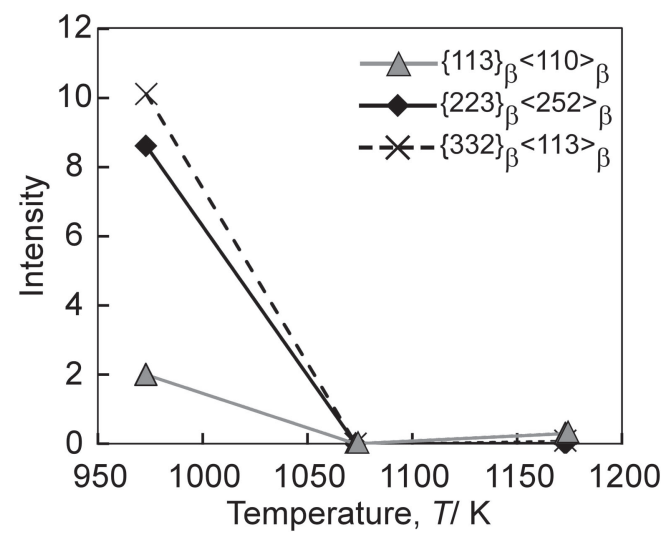

Fig. 8 Heat treatment temperature dependence of the texture strength of (a) Goss, $\{110\}_{\beta}\langle 113\rangle_{\beta}$, and Brass orientations, (b) $\{113\}_{\beta}\langle 110\rangle_{\beta}$, $\{223\}_{\beta}\langle 252\rangle_{\beta}$, and $\{332\}_{\beta}\langle 113\rangle_{\beta}$.

$0.4 \mu \mathrm{m}$ and $33 \%$, respectively. Figure 10 shows the heat treatment temperature dependence of the $\beta$ grain size: the grain size decreased with decreasing heat treatment temperature. In particular, the microstructure in HT973 was extremely fine, and the $\beta$ grain size decreased from $60.8 \mu \mathrm{m}$ to $0.5 \mu \mathrm{m}$ with a decrease in the heat treatment temperature from $1073 \mathrm{~K}$ to $973 \mathrm{~K}$. It has been reported that secondary recrystallization is responsible for the development of the Goss orientation as the preferred orientation in grain-oriented silicon steels. ${ }^{33)}$ However, in the present study, no large 

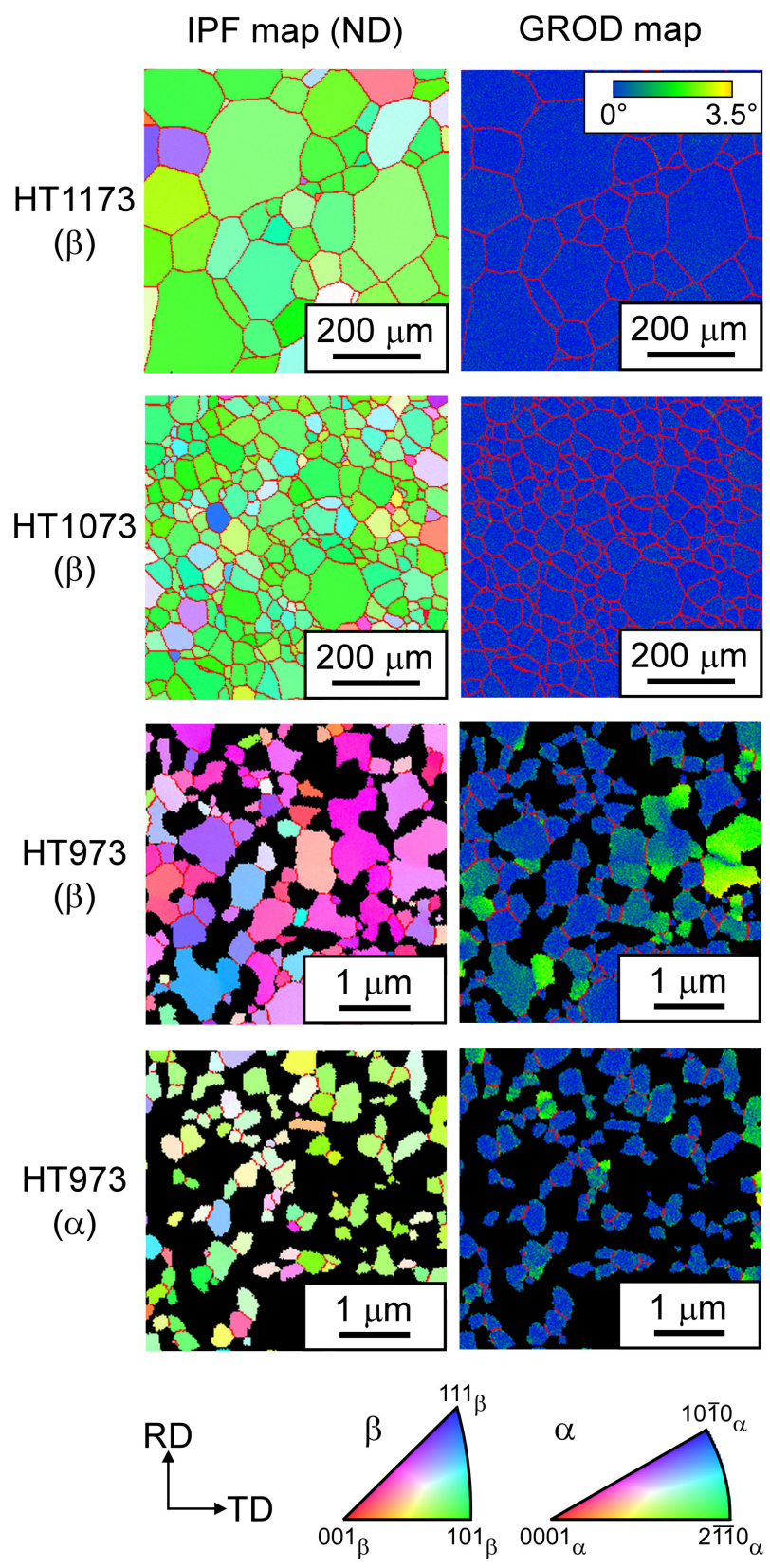

Fig. 9 IPF (ND) and GROD maps for HT1173, HT1073 and HT973 specimens. Grain boundaries are marked in red.

grains were observed, regardless of the heat treatment temperature. The average sizes of the Goss-oriented grains in HT1173 and HT1073 are 140.3 and $77.2 \mu \mathrm{m}$, respectively. The average sizes of the grains not showing the Goss, $\{110\}_{\beta}\langle 113\rangle_{\beta}$, and Brass orientations (that is, orientations not formed as a texture component in HT1173 and HT1073) are 132.3 and $50.3 \mu \mathrm{m}$, respectively.

GROD maps, which are indicators of strain in the crystal map, are shown in the right column of Fig. 9. These are shaded according to the angular deviation to the reference orientation for the grain to which the point corresponds to. High GROD grains $\left(>2^{\circ}\right)$ of the $\alpha$ and $\beta$ phase exist in the GROD maps for HT973, while the grains in HT1173 and HT1073 show a low GROD. The high GROD in the case of HT973 is thought to be associated with the high density of dislocations induced by cold-rolling.

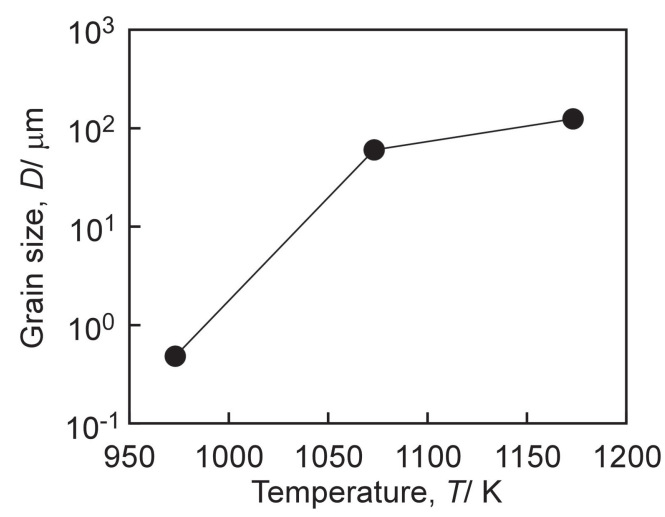

Fig. 10 Heat treatment temperature dependence of the grain size of $\beta$ phase.

The texture and microstructure (grain size and GROD) in HT973 appear to be different from those of HT1173 and HT1073, therefore the microstructure in HT973 was investigated in detail by TEM observations. Figures 11(a) and (b) show the bright-field (BF) image and selected-area diffraction pattern (SADP) of HT973. The key diagram of the $\mathrm{SADP}$ is shown in Fig. 11(c). Fine grains and a Debye ringlike pattern of the $\alpha$ and $\beta$ phases were observed. In addition, a contrast of the dislocations induced by cold-rolling was observed, and the specimen was not recrystallized completely. A dark-field (DF) image of the $\alpha$ phase is shown in Fig. 11(d). The DF image was formed using the reflection indicated by the thick circle in Fig. 11(b). The globular $\alpha$ phase was observed to exist at the grain boundary. These results were consistent with those of the EBSD analysis.

Texture of $\beta$ phase in HT973 is considered to be formed by recrystallization and/or reverse transformation from $\alpha^{\prime \prime}$ to $\beta$ (and recovery) because HT973 was not recrystallized completely. Especially, it is likely that $\{113\}_{\beta}\langle 110\rangle_{\beta}$ is not formed by reverse transformation because all the orientations of $\alpha^{\prime \prime}$ that correspond to $\{113\}_{\beta}\langle 110\rangle_{\beta}$ do not satisfy the condition of candidate textures in the CR specimen mentioned in section 3.2: $(142)_{\alpha^{\prime \prime}}[2 \overline{1} 1]_{\alpha^{\prime \prime}},(\overline{1} 24)_{\alpha^{\prime \prime}}[\overline{2} 1 \overline{1}]_{\alpha^{\prime \prime}}$, $(14 \overline{2})_{\alpha^{\prime \prime}}[\overline{2} 11]_{\alpha^{\prime \prime}}, \quad(\overline{1} 24)_{\alpha^{\prime \prime}}[211]_{\alpha^{\prime \prime}}, \quad(320)_{\alpha^{\prime \prime}}[00 \overline{1}]_{\alpha^{\prime \prime}}, \quad$ and $(\overline{302})_{\alpha^{\prime \prime}}[0 \overline{1} 0]_{\alpha^{\prime \prime}}$ correspond to $(113)_{\beta}[1 \overline{1} 0]_{\beta}$. The definition of those six lattice correspondence variants is given in Ref. 21). The results of microstructure observation also imply that complete recrystallization of the specimen is critical for Goss orientation formation in the Ti-5.5Mo-8Al$6 \mathrm{Zr}$ alloy. In previous study, two mechanisms are proposed to understand the change in orientation by $\alpha$ phase precipitation. One is the possibility of $\alpha$ phase precipitation in the highly strained region, and the other case is the pinning of particular grain boundaries especially the grain boundary of the Goss, $\{110\}_{\beta}\langle 113\rangle_{\beta}$, and Brass oriented grains. ${ }^{23)}$ Highly strained regions such as shear bands and grain boundaries are preferential nucleation sites for precipitation and the initiation sites for recrystallization. When $\alpha$ phase precipitation at these preferential sites occurs before recrystallization, the recrystallization behavior is strongly affected by the precipitation in the present alloy. The formation mechanism of the $\alpha$ phase and the effect of the $\alpha$ phase on the recrystallization texture are unclear, however, TEM observation results in this study support the mechanism proposed. 

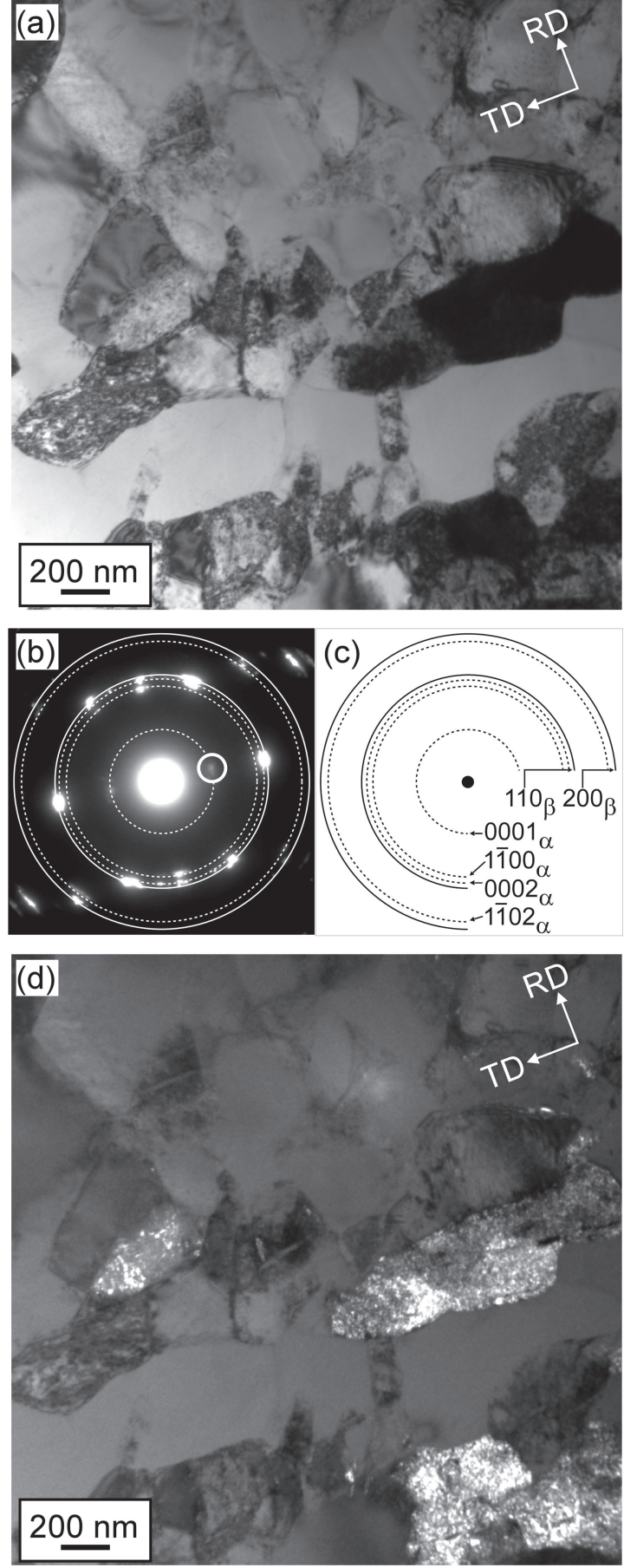

Fig. 11 (a) A BF image and (b) SADP of HT973. (c) Key diagram of the SADP. Thin solid and broken lines in (b) and (c) indicate reflections from $\beta$ and $\alpha$ phases, respectively. (d) A DF image taken at the $\alpha$ phase diffraction spot indicated by the thick circle in (b).

\section{Conclusion}

The effect of the heat treatment temperature on the evolution of the recrystallization texture was investigated in cold-rolled $\mathrm{Ti}-5.5 \mathrm{Mo}-8 \mathrm{Al}-6 \mathrm{Zr}(\mathrm{mol} \%)$ alloy sheets and the following conclusions were drawn:

(1) $\alpha^{\prime \prime}$ martensite was induced by cold-rolling. The deformation texture of $\alpha^{\prime \prime}$ martensite could not be detected, but the formation of $\{130\}_{\alpha^{\prime \prime}}\langle 3 \overline{1} 0\rangle_{\alpha^{\prime \prime}}$ and $(100)_{\alpha^{\prime \prime}}[010]_{\alpha^{\prime \prime}}$ textures was proposed.
(2) The specimens heat-treated at $1073 \mathrm{~K}$ or higher consisted of a single $\beta$ phase. Goss, $\{110\}_{\beta}\langle 113\rangle_{\beta}$, and Brass orientations were formed as the recrystallization textures in these specimens. In particular, the Goss orientation strongly developed with increasing heat treatment temperature and became the dominant component in the specimens heat-treated at $1173 \mathrm{~K}$.

(3) The specimen heat-treated at $973 \mathrm{~K}$ consisted of the $\beta+\alpha$ phase. $\{113\}_{\beta}\langle 110\rangle_{\beta}, \quad\{113\}_{\beta}\langle 141\rangle_{\beta}$, $\{223\}_{\beta}\langle 252\rangle_{\beta},\{223\}_{\beta}\langle 692\rangle_{\beta}$, and $\{332\}_{\beta}\langle 113\rangle_{\beta}$ were formed in this specimen, and the texture components observed in the specimens heat-treated at $1073 \mathrm{~K}$ or higher vanished. The $\alpha$ phase formed $\{11 \overline{2} 0\}_{\alpha}\langle 1 \overline{1} 00\rangle_{\alpha}-$ $\{11 \overline{2} 2\}_{\alpha}\langle 1 \overline{1} 00\rangle_{\alpha}$. TEM observations revealed that the specimen heat-treated at $973 \mathrm{~K}$ was not completely recrystallized.

\section{Acknowledgments}

This work was supported by the Japan Society for the Promotion of Science (Grant numbers Wakate B: 17K14833, Wakate A: 17H04959, Kiban S: 26220907, and Kiban B: 18H01728).

\section{REFERENCES}

1) M. Niinomi: Metall. Mater. Trans. A 33 (2002) 477-486

2) M. Niinomi: Sci. Technol. Adv. Mater. 4 (2003) 445-454.

3) W. Haider, N. Munroe, C. Pulletikurthi, P.K.S. Gill and S. Amruthaluri: J. Mater. Eng. Perform. 18 (2009) 760-764.

4) T. Inamura, Y. Kinoshita, J.I. Kim, H.Y. Kim, H. Hosoda, K. Wakashima and S. Miyazaki: Mater. Sci. Eng. A 438-440 (2006) 865-869.

5) T. Inamura, Y. Yamamoto, H. Hosoda, H.Y. Kim and S. Miyazaki: Acta Mater. 58 (2010) 2535-2544.

6) H.Y. Kim, T. Sasaki, K. Okutsu, J.I. Kim, T. Inamura, H. Hosoda and S. Miyazaki: Acta Mater. 54 (2006) 423-433.

7) L. Wang, W. Lu, J. Qin, F. Zhang and D. Zhang: Mater. Charact. 61 (2010) 535-541.

8) M.F. Ijaz, H.Y. Kim, H. Hosoda and S. Miyazaki: Mater. Sci. Eng. C 48 (2015) 11-20.

9) L.L. Pavón, H.Y. Kim, H. Hosoda and S. Miyazaki: Scr. Mater. 95 (2015) 46-49.

10) Y. Shinohara, M. Tahara, T. Inamura and H. Hosoda: J. Japan Inst. Met. Mater. 80 (2015) 45-50.

11) J. Fu, H.Y. Kim and S. Miyazaki: J. Mech. Behav. Biomed. Mater. 65 (2017) 716-723.

12) T. Inamura, R. Shimizu, H.Y. Kim, S. Miyazaki and H. Hosoda: Mater. Sci. Eng. C 61 (2016) 499-505.

13) H. Tobe, H.Y. Kim and S. Miyazaki: J. Japan Inst. Metals 72 (2008) 965-969.

14) F.W. Ling, E.A. Starke and B.G. Lefevre: Metall. Trans. 5 (1974) 179187.

15) J. Fu, A. Yamamoto, H.Y. Kim, H. Hosoda and S. Miyazaki: Acta Biomater. 17 (2015) 56-67.

16) H.Y. Kim, T. Fukushima, P.J.S. Buenconsejo, T.-h. Nam and S. Miyazaki: Mater. Sci. Eng. A 528 (2011) 7238-7246.

17) H.Y. Kim, N. Oshika, J.I. Kim, T. Inamura, H. Hosoda and S. Miyazaki: Mater. Trans. 48 (2007) 400-406.

18) H.Y. Kim, Y. Ikehara, J.I. Kim, H. Hosoda and S. Miyazaki: Acta Mater. 54 (2006) 2419-2429.

19) H.Y. Kim, J. Fu, H. Tobe, J.I. Kim and S. Miyazaki: Shap. Mem. Superelasticity 1 (2015) 107-116.

20) L.L. Pavón, E.L. Cuellar, S.V. Hernandez, I.E. Moreno-Cortez, H.Y. Kim and S. Miyazaki: J. Alloys Compd. 782 (2019) 893-898.

21) M. Tahara, N. Okano, T. Inamura and H. Hosoda: Sci. Rep. 7 (2017) 15715 . 
22) K. Hiramatsu, M. Tahara, T. Inamura, H. Hosoda and S. Miyazaki: Mater. Sci. Forum 738-739 (2013) 262-266.

23) T. Sasaki, K. Hiramatsu, M. Tahara, H. Hosoda, S. Miyazaki and T Inamura: Adv. Mater. Res. 922 (2014) 622-625.

24) Y. Shinohara, D. Narita, M. Tahara, H. Hosoda and T. Inamura: Mater Trans. 57 (2016) 1998-2001.

25) B. Sander and D. Raabe: Mater. Sci. Eng. A 479 (2008) 236-247.

26) T. Inamura, Y. Fukui, H. Hosoda, K. Wakashima and S. Miyazaki: Mater. Trans. 45 (2004) 1083-1089.

27) M. Tane, S. Akita, T. Nakano, K. Hagihara, Y. Umakoshi, M. Niinomi and H. Nakajima: Acta Mater. 56 (2008) 2856-2863.

28) Y. Shinohara, Y. Matsumoto, M. Tahara, H. Hosoda and T. Inamura: Materialia 1 (2018) 52-61.

29) T. Inamura, H. Hosoda, K. Wakashima and S. Miyazaki: Mater. Trans. 46 (2005) 1597-1603.

30) Z. Guo, S. Malinov and W. Sha: Comput. Mater. Sci. 32 (2005) 1-12.

31) H. Hu: Texture 1 (1974) 233-258.

32) W.G. Burgers: Physica 1 (1934) 561-586.

33) Z. Xia, Y. Kang and Q. Wang: J. Magn. Magn. Mater. 320 (2008) 3229-3233. 